\title{
Thymoma Presenting as Right-Side Heart Failure in a Young Healthy Patient: A Case Report and Literature Review
}

\author{
Rabih E. Tabet ${ }^{\mathrm{a}, \mathrm{f}}$, Mahmoud Hussein ${ }^{\mathrm{a}}$, Boutros Karam ${ }^{\mathrm{a}}$, Tanios Otayek ${ }^{\mathrm{b}}$, Elie F. Akoury ${ }^{\mathrm{c}}$, \\ Georges Nasrallah ${ }^{\mathrm{d}}$, Sarkis Ejbeh ${ }^{\mathrm{e}}$
}

\begin{abstract}
Thymoma is a rare tumor yet the most common neoplasm of the anterior mediastinum, accounting for $20-25 \%$ of all mediastinal tumors. Its incidence peaks in the fourth and fifth decades of life. Usually thymoma is diagnosed incidentally on chest imaging, or when investigating patients with myasthenia gravis, or with chest symptoms such as cough, chest pain, or dyspnea. We report here the case of a 31-year-old male patient with a swinging heart due to thymoma who presented with abdominal ascites and lower limbs edema, treated successfully with surgery and radiotherapy. This is, to our knowledge, the first case of thymoma to present this way with right-side heart failure like symptoms.
\end{abstract}

Keywords: Anterior mediastinal mass; Thymoma; Swinging heart; Massive pericardial effusion; Ascites

\section{Introduction}

Thymoma is an uncommon tumor originating from the epithelial cells of the thymus [1]. It is best known for its association with myasthenia gravis (MG) which occurs in about $50 \%$ of patients with thymoma. Most cases occur between the ages of 40 and 60 years [2] with a mean age at presentation being 52 years. Thymoma may present in three main different ways: $30-50 \%$ of patients present with an asymptomatic an-

Manuscript accepted for publication March 31, 2016

${ }^{a}$ Department of Cardiovascular Medicine, Faculty of Medical Sciences, The Lebanese University, Beirut, Lebanon

${ }^{b}$ Department of Internal Medicine, Our Lady of Lebanon University Hospital, Jounieh, Lebanon

'Department of Cardiovascular Medicine, Our Lady of Lebanon University Hospital, Jounieh, Lebanon

${ }^{\mathrm{d} D e p a r t m e n t ~ o f ~ C a r d i a c ~ A n e s t h e s i a ~ a n d ~ t h e ~ C a r d i a c ~ S u r g e r y ~ U n i t, ~ O u r ~ L a d y ~ o f ~}$ Lebanon University Hospital, Jounieh, Lebanon

eDepartment of Cardiothoracic Surgery, Our Lady of Lebanon University Hospital, Jounieh, Lebanon

${ }^{f}$ Corresponding Author: Rabih Tabet, Department of Cardiovascular Medicine, Faculty of Medical Sciences, The Lebanese University, Hadath, Beirut, Lebanon. Email: drabih_tabet@hotmail.com

doi: http://dx.doi.org/10.14740/jmc2478e terior mediastinal mass on chest roentgenogram. Around onethird of patients are diagnosed during the evaluation of MG or other paraneoplastic syndromes such as pure red cell aplasia or acquired hypogammaglobulinemia [3], and one-third of cases present with local symptoms such as cough, superior vena cava syndrome, chest pain, or dysphagia among others [1]. This report describes a case of thymoma in a young male patient who presented with a picture of right-side heart failure with ascites and lower limbs edema and was found to have a swinging heart and was treated successfully with surgery and radiotherapy. This is an unusual presentation of thymoma that does not fit in any of the three categories described above.

\section{Case Report}

In September 2015, a 31-year-old man came to our institution because of increasing abdominal girth, lower limbs edema, and exertional dyspnea. The patient's symptoms started 2 weeks prior, with a progressive increase in belly size and body weight. Next, he noticed bilateral feet edema, and then started having shortness of breath upon exertion. In addition, the patient's bowel movements decreased to fewer than once every 2 days. It is worth noting that the patient had no previous medical history except for a resolved hepatitis A infection 10 years prior.

At his presentation, the patient was in no respiratory distress, complaining only of abdominal distension and lower limbs edema. His vital signs were within normal range except for mild tachycardia (heart rate around $106 \mathrm{bpm}$ ). The blood pressure was maintained around 126/82 mm Hg. On physical examination, he had jugular venous distension and decreased breath sounds upon auscultation of the lower lung fields. The heart sounds were soft without any additional murmur. His abdomen was distended. Peri-umbilical tympanism and peripheral dullness were present upon percussion. Also, shifting dullness was noted when turning the patient on his side. Finally, he had bilateral lower limbs pitting edema reaching his mid-legs. Laboratory tests returned unremarkable with normal cells count, normal chemistry, and biology. Even the inflammatory markers were non-significant (CRP of $5 \mathrm{mg} / \mathrm{L}$ and an ESR of $15 \mathrm{~mm} / \mathrm{h}$ ). Ultrasound examination of the abdomen showed moderate ascites, and the peritoneal paracentesis showed a transudate nature of the fluid. Electrocardiogram (Fig. 1) showed microvoltage with sinus tachycardia and chest roentgenogram (Fig. 1) revealed cardiomegaly with bilateral 


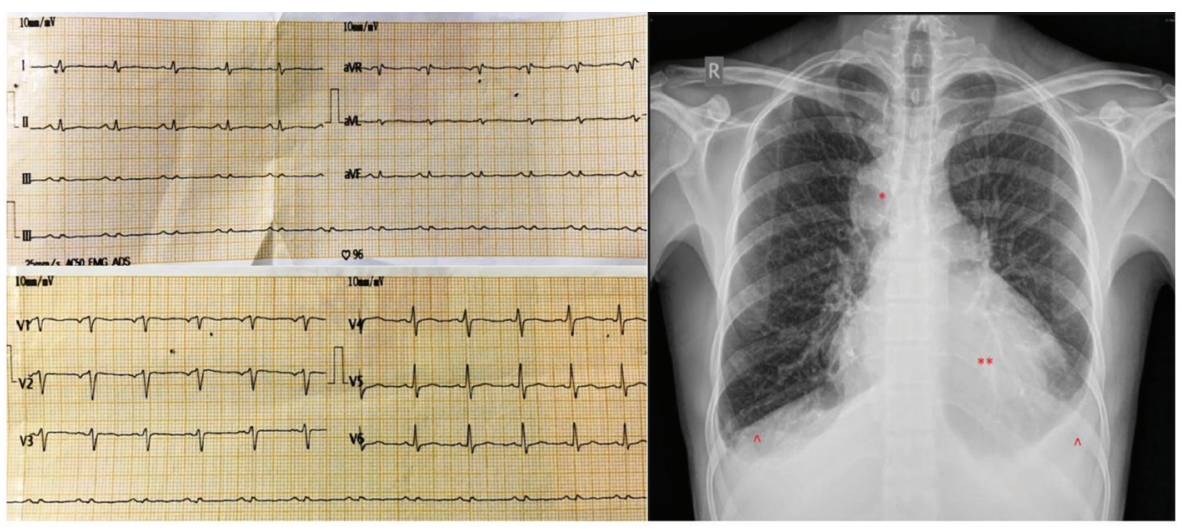

Figure 1. (Left) Electrocardiogram upon presentation showing microvoltage with sinus tachycardia. (Right) Chest roentgenogram upon presentation showing cardiomegaly $\left({ }^{* *}\right)$ with bilateral pleural effusions $\left({ }^{\wedge}\right)$ and a right upper paramediastinal round opacity $\left({ }^{*}\right)$.

pleural effusions and a right upper parasternal round opacity. Upon these results, and to further investigate the cause of his transudative ascites, a transthoracic echocardiography and a computed tomography scan of the chest, abdomen and pelvis were scheduled. Echocardiography showed a swinging heart in a massive circumferential pericardial effusion (Fig. 2), so the patient was transferred emergently to the operating room and a pericardial window with drainage of the pericardial fluid was done. The pericardial fluid was bloody (numerous red blood cells were seen), containing 5,300 white blood cells per $\mathrm{mm}^{3}$ with $80 \%$ lymphocytes; LDH level was $1,632 \mathrm{U} / \mathrm{L}$ (for a serum LDH level of $138 \mathrm{IU} / \mathrm{L}$ ), with a glucose level of $0.7 \mathrm{~g} / \mathrm{L}$ and proteins level of $4.7 \mathrm{~g} / \mathrm{dL}$ (for a serum proteins level of $6.2 \mathrm{~g} / \mathrm{dL}$ ). It was considered as an exudate according to Light's criteria. Pericardial fluid culture returned sterile and the cytopathology study showed no malignant cells. Pericardial biopsy only showed abundant pericapillary mononuclear cells infiltrate (non-specific vasculitis). The computed tomography scan showed a supracardiac, retrosternal irregular mass $(69 \times 46 \mathrm{~mm}$ in size $)$ repulsing the ascending aorta and the superior veina cava posteriorly, in continuity with a smaller round mass $(47 \times 45 \mathrm{~mm}$ in size $)$ in the right para-tracheal

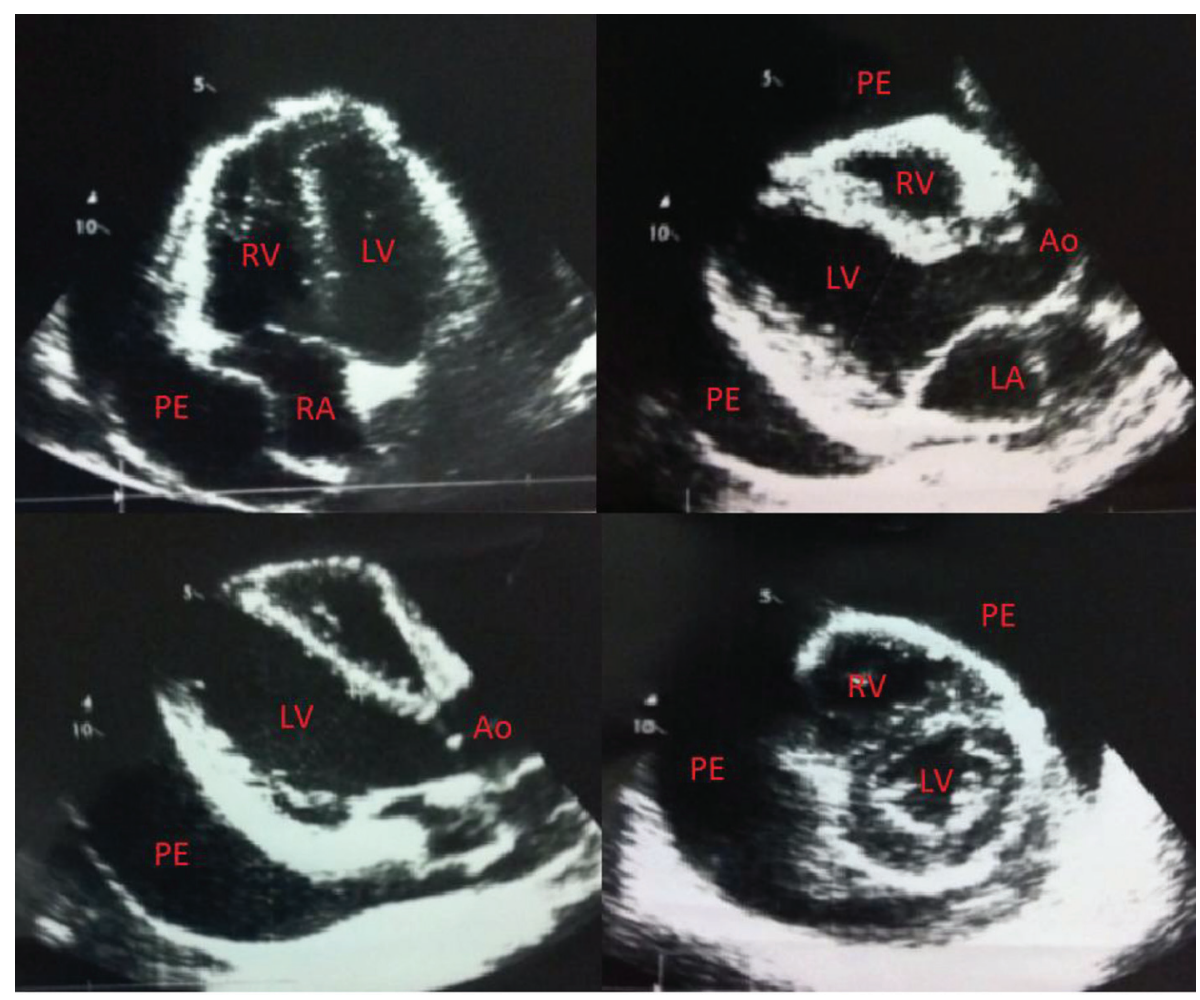

Figure 2. Echocardiogram showing a swinging heart in a massive circumferential pericardial effusion. LV: left ventricle; RV: right ventricle; LA: left atrium; RA: right atrium; Ao: aorta; PE: pericardial effusion. 


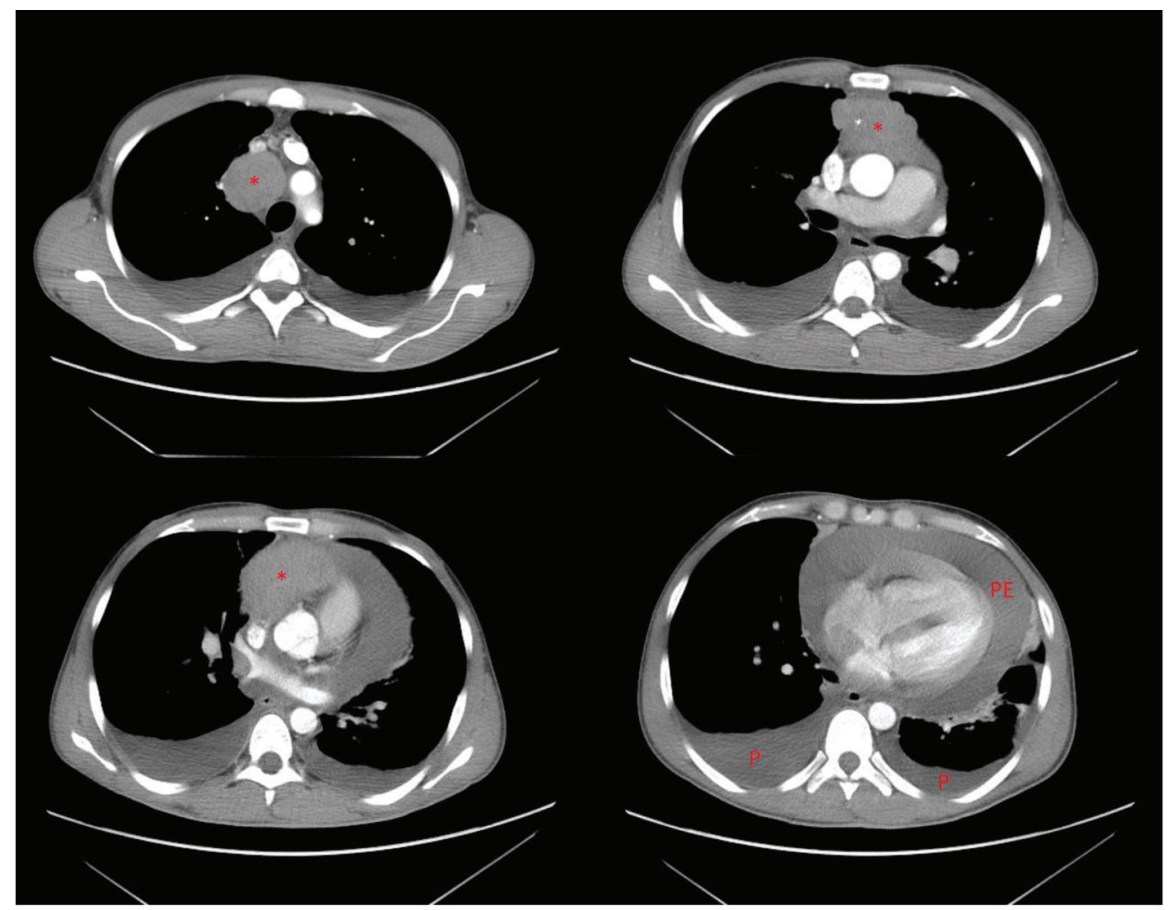

Figure 3. Computed tomography scan of the chest showing a retrosternal mass $\left(^{*}\right)$ with massive pericardial effusion $(\mathrm{PE})$ and bilateral pleural effusions $(P)$.

area (Fig. 3). Beta human chorionic gonadotropin (BHCG) and alpha-fetoprotein (AFP) levels were ordered as investigations of this anterior mediastinal mass but returned negative (BHCG level of $0 \mathrm{mIU} / \mathrm{mL}$, AFP level of $0.605 \mathrm{ng} / \mathrm{mL}$ ). The patient then was scheduled for a right mediastinotomy (Chamberlaine's approach) and biopsy of the mass that revealed a tumoral proliferation composed of lymphocytic elements intercepted by epithelial cells bands in favor of a lympho-epithelial thymoma. Immunohistochemistry studies confirmed the diagnosis with a positivity of anti-pan cytokeratin, anti-LCA, and anti-CD1a antibodies. The patient was then evaluated for MG with acetyl-choline receptors antibodies level and MusK antibodies level which returned negative (anti-AChr antibodies level of $0.1 \mathrm{nmol} / \mathrm{L}$; anti-musk antibodies level of $0.01 \mathrm{U} /$ $\mathrm{mL}$ ). He was later on scheduled for sternotomy and total wide resection of the mass, including pericardial sac excision and bilateral apical lungs resections at the site of the macroscopic intra-operative invasion. The definitive pathological study of the tumor established the staging of the disease by showing a lympho-epithelial thymoma with atypical and aggressive cells, invading the pericardium, the pleura and the adjacent lung tissue, and since the whole tumor was resected with free margins on the pathology, the staging was set to be Masaoka classification stage III and GETT (Groupe d'Etudes des Tumeurs Thymiques) classification stage II.

\section{Discussion}

Thymomas are very rare neoplasms that arise from thymic tissue elements. Thymomas are slow-growing tumors, yet, they are considered to be malignant because of their potential invasiveness. Note that invasion beyond the capsule is a major prognostic factor that correlates with a worse outcome. Surgery with radical excision of the tumor is usually the first step in the management of thymomas, completed with radiotherapy and chemotherapy according to the stage of the disease [4].

Thymomas typically present in one of three major ways: 1) as an incidental finding on thoracic imaging in an asymptomatic patient, or 2) because of local (thoracic) symptoms and their subsequent evaluation (such as chest pain, cough, shortness of breath, and phrenic nerve palsy among others), or 3) during the evaluation of a paraneoplastic syndrome (such as $\mathrm{MG}$, pure red cell aplasia, and hypogammaglobulinemia among others) [5]. Three observations are worth-noting in our case report. First, the age of the patient (31-year-old) is remarkable, because most cases of thymoma occur between the fourth and fifth decades of life $[6,7]$. Second, the patient had none of the usual presenting symptoms of thymoma neither had he the symptoms of MG [8]. Instead, he presented with abdominal ascites, lower limbs edema and jugular venous distension, resembling the presentation of a right-side heart failure, which is to our knowledge the first case to present this way. Third, the patient had a massive pericardial effusion with a swinging heart; however, it remained silent without causing any shortness of breath, nor cough or chest pain. This is most likely due to the slow rate of accumulation of the pericardial effusion. The possible mechanism of formation of the pericardial effusion is the disordered lymphatic return caused by the tumor growth. Another mechanism may be the inflammatory reaction caused by tumor cells [9], and in this case, the pericardial volume may reach $2 \mathrm{~L}$ before a critical life-threatening 
Table 1. WHO Histologic Typing of Tumors of the Thymus

\begin{tabular}{llll}
\hline Type & & Characteristics & Frequency \\
\hline Type A & Medullary thymoma & Spindle cells; medullary & $9 \%$ \\
Type AB & Mixed thymoma & Mixed cellularity & $24 \%$ \\
Type B1 & Predominantly cortical thymoma & Lymphocyte-rich & $13 \%$ \\
Type B2 & Cortical thymoma & Predominantly lymphocytic & $24 \%$ \\
Type B3 & Well differentiated thymic carcinoma & Epithelial cells with mild atypia & $15 \%$ \\
Type C & Thymic carcinoma & Highly atypical cells & $15 \%$ \\
\hline
\end{tabular}

tamponade occurs [10].

A patient with suspected thymoma should be initially evaluated by a thoracic imaging modality (computed tomography scan and/or magnetic resonance imaging), which establishes the presence of an anterior mediastinal mass and delivers the initial information about the resectability of the tumor. The differential diagnosis includes retrosternal thyroid, lymphoma, and mediastinal germ cell tumor. Thus, preoperative assessment may include germ cell tumor markers (BHCG and AFP) and thyroid function tests.

The most widely used system to classify thymic neoplasms based upon their histologic and pathologic appearance is that of the World Health Organization (WHO). The cellular composition of thymomas correlates very well with the behavior of the tumor and with its prognosis [11]. The subsets of primary thymic tumors are divided as shown in Table 1 $[12,13]$.

In addition to the histologic type of the tumor, the stage of thymoma correlates greatly with the survival of the patient [14-16]. The most widely used system to stage both thymomas and thymic carcinomas is the Masaoka staging system which is based upon the extent of the primary tumor and the presence of invasion into adjacent structures and/or dissemination (Table 2) $[13,17]$.

The definitive diagnosis of a thymoma requires a tissue diagnosis. For patients thought to have a tumor that is amenable to complete resection, the initial step in management is surgical resection, which will establish the diagnosis and provide the primary treatment. In contrast, for patients who have a disease that is not considered amenable to complete resection or for whom surgery is contraindicated for any reason, a tissue diagnosis using either a core biopsy or an open biopsy is required prior to therapy $[13,17]$.

Nowadays, there is still a lack of randomized controlled trials that provide guidance for the management of patients with thymoma due to the rarity of these tumors. Treatment guidelines have been promulgated based on data from retrospective series only $[13,17]$. These guidelines rely on the results of the postoperative staging and they may be resumed as follow. For patients with stage I or stage IIA, no postoperative adjuvant treatment is indicated (neither radiotherapy nor chemotherapy). For patients with stage IIB disease, treatment with radiotherapy should follow the surgery. Patients with potentially resectable stage III or IVA tumors should receive postoperative radiotherapy and/or chemotherapy. Lastly, patients with unresectable stage III or IV tumors or patients who are medically inoperable may benefit from systemic chemotherapy $[13,17]$. To note that the presence of invasion is an important adverse prognostic marker $[1,18]$ and the likelihood of long-term survival depends upon the completeness of surgical resection $[19,20]$. Sometimes the resection of the pericardium as well as accompanying lung parenchyma is required to achieve a complete resection with histologically negative margins [21], as it was the case of our patient. Although there are no clinical trials providing evidence of benefit, monitoring for recurrences with thoracic imaging on an annual basis is warranted given that early intervention may be more effective or at least more feasible [22].

The patient in our case report had a stage IIIA thymoma. The whole tumor was resected and the final pathology showed disease-free margins, and thus he was scheduled for radiotherapy to complete his treatment as recommended for this stage of the disease. At this time, he is undergoing radiotherapy sessions which he is tolerating well. A computed tomography

Table 2. Masaoka Staging System for Thymoma and Thymic Carcinoma

\begin{tabular}{lll}
\hline Stage & Characteristic & 5-year overall survival \\
\hline Stage I & Macroscopically and microscopically completely encapsulated & $94-100 \%$ \\
Stage IIA & Microscopic transcapsular invasion & $86-95 \%$ \\
Stage IIB & $\begin{array}{l}\text { Macroscopic invasion into surrounding fatty tissue or grossly adherent to but not } \\
\text { through the mediastinal pleura or pericardium }\end{array}$ & $86-95 \%$ \\
Stage IIIA & Macroscopic invasion into pericardium or lung without great vessel invasion & $56-69 \%$ \\
Stage IIIB & Macroscopic invasion into pericardium or lung with great vessel invasion & $56-69 \%$ \\
Stage IVA & Pleural or pericardial dissemination & $11-50 \%$ \\
Stage IVB & Lymphogenous or hematogenous metastases & $11-50 \%$ \\
\hline
\end{tabular}


scan of the chest will be scheduled later on to monitor any recurrences.

\section{Conclusion}

Thymomas are very rare tumors with a very wide spectrum of presentations. Early recognition and diagnosis with timely and complete resection are essential for a better prognosis and higher survival rate.

\section{Financial Support}

No financial support was given for this publication.

\section{Conflicts of Interest}

None of the authors has any conflict of interest.

\section{Abbreviations}

MG: myasthenia gravis; BHCG: beta human chorionic gonadotropin; AFP: alpha-fetoprotein; WHO: World Health Organization

\section{References}

1. Thomas CR, Wright CD, Loehrer PJ. Thymoma: state of the art. J Clin Oncol. 1999;17(7):2280-2289.

2. Kallas E, Hueb A, Kallas I, Kallas A. Middle mediastinal thymoma: case report. Braz J Cardiovasc Surg. 2005;20(2):189-191.

3. Hamidah A, Poulsaeman V, Suria AA, Zarina AL, Sulfiqar MA, Jamal R. Thymoma: A case report and review of the literature. Med \& Health. 2010;5(1):49-54.

4. Cowen D, Hannoun-Levi JM, Resbeut M, Alzieu C. Natural history and treatment of malignant thymoma. Oncology (Williston Park). 1998;12(7):1001-1005; discussion 1006.

5. Kendrix E, A Letch K, Quintessa M. Thymoma. Medscape. December 16, 2015 update.

6. Mullen B, Richardson JD. Primary anterior mediastinal tumors in children and adults. Ann Thorac Surg. 1986;42(3):338-345.

7. Wilkins EW, Jr., Grillo HC, Scannell JG, Moncure AC, Mathisen DJ. J. Maxwell Chamberlain Memorial Paper. Role of staging in prognosis and management of thymoma. Ann Thorac Surg. 1991;51(6):888-892.
8. Cheng MF, Tsai CS, Chiang PC, Lee HS. Cardiac tamponade as manifestation of advanced thymic carcinoma. Heart Lung. 2005;34(2):136-141.

9. Nishi T, Takamori S, Muta F, Yoshiyama K, Iwasaki Y, Shirouzu K. Nonmalignant pericardial effusion associated with thymic cancer. Gen Thorac Cardiovasc Surg. 2010;58(5):239-242.

10. Spodick DH. Acute cardiac tamponade. N Engl J Med. 2003;349(7):684-690.

11. Travis WD, Brambilla E, Muller-Hermelink HK, Harris CC. Pathology \& genetics: Tumours of the lung, pleura, thymus and heart. World Health Organization Classification of tumours, IARC Press, Lyon, France 2004.

12. Detterbeck FC. Clinical value of the WHO classification system of thymoma. Ann Thorac Surg. 2006;81(6):23282334.

13. Falkson CB, Bezjak A, Darling G, Gregg R, Malthaner R, Maziak DE, Yu E, et al. The management of thymoma: a systematic review and practice guideline. J Thorac Oncol. 2009;4(7):911-919.

14. Masaoka A, Monden Y, Nakahara K, Tanioka T. Followup study of thymomas with special reference to their clinical stages. Cancer. 1981;48(11):2485-2492.

15. Schneider PM, Fellbaum C, Fink U, Bollschweiler E, Prauer HW. Prognostic importance of histomorphologic subclassification for epithelial thymic tumors. Ann Surg Oncol. 1997;4(1):46-56.

16. Kim DJ, Yang WI, Choi SS, Kim KD, Chung KY. Prognostic and clinical relevance of the World Health Organization schema for the classification of thymic epithelial tumors: a clinicopathologic study of 108 patients and literature review. Chest. 2005;127(3):755-761.

17. National Comprehensive Cancer Network guidelines file://www.nccn.org/professionals/physician_gls/f_ guidelines.asp (Accessed on April 17, 2012).

18. Gripp S, Hilgers K, Wurm R, Schmitt G. Thymoma: prognostic factors and treatment outcomes. Cancer. 1998;83(8):1495-1503.

19. Strobel P, Bauer A, Puppe B, Kraushaar T, Krein A, Toyka K, Gold R, et al. Tumor recurrence and survival in patients treated for thymomas and thymic squamous cell carcinomas: a retrospective analysis. J Clin Oncol. 2004;22(8):1501-1509.

20. Davenport E, Malthaner RA. The role of surgery in the management of thymoma: a systematic review. Ann Thorac Surg. 2008;86(2):673-684.

21. Maggi G, Casadio C, Cavallo A, Cianci R, Molinatti M, Ruffini E. Thymoma: results of 241 operated cases. Ann Thorac Surg. 1991;51(1):152-156.

22. NCCN Clinical Practice Guidelines in Oncology. Thymic Malignancies. V.2.2010. file://www.nccn.org/professionals/physician_gls/PDF/thymic.pdf. 